\title{
EFFECT OF SMALL HAIRPIN RNA MOLECULES TARGETING ENDOTHELIN- CONVERTING-ENZYME-1 IN MONOCROTALINE-INDUCED PULMONARY HYPERTENSIVE RATS
}

\author{
Y.M. Hong ${ }^{1}$, J.S. Son ${ }^{2}$, K.C. Kim ${ }^{3}$ \\ ${ }^{1}$ Pediatrics, Ewha Womans University, ${ }^{2}$ Pediatrics, Konkuk University, ${ }^{3}$ Thoracic and Cardiovascular \\ Surgery, Ewha Womans University, Seoul, Republic of Korea
}

Purpose: The purpose of this study was to investigate the therapeutic effects of an small hairpin RNA (shRNA) molecules targeting endothelin converting enzyme-1 (ECE-1) in monocrotaline(MCT)-induced pulmonary hypertension in rats.

Methods: Sprague-Dawley Rats were treated with normal saline (control group), with MCT (60mg/kg subcutaneous injection, MCT group), and with MCT + recombinant lentiviral vectors carrying shRNA targeting ECE-1 (shRNA group). To assess the effects of shRNA targeting ECE-1 on the time course.

Results: MCT group showed a marked increase in mean RVP compared to control group on days 4, 7, 14 and 28 . The shRNA group showed a significant improvement in mean RVP days on 4, 7, 14 and 28. The serum concentration of ET-1 was significantly lower in shRNA group. The expression of ECE-1, ET (endothelin)-1 and ETA receptor of the lung were significantly increased in MCT group on days 14 and 28. The expression of ETA receptor was significantly decreased in shRNA group on day 14. Quantitative analysis of peripheral pulmonary arteries revealed that medial wall thickness was significantly increased after MCT injection, and significantly decreased in shRNA group on days 14 and 28 . The number of intraacinar muscular arteries after MCT injection was significantly decreased in shRNA group on day 14 .

Conclusions: Recombinant lentiviral vectors carrying shRNA targeting ECE-1 prevented RVP and RV hypertrophy, exerted an anti-proliferative effect on pulmonary artery smooth muscle cells. Our results indicate that this treatment modality is a promising that could protect against hemodynamic and histopathological changes in pulmonary hypertension. 